CUQM-124

\title{
The Klein-Gordon equation with the Kratzer potential in $d$ dimensions
}

\author{
Nasser Saad* \\ Department of Mathematics and Statistics, University of Prince Edward Island, \\ 550 University Avenue, Charlottetown, PEI, Canada C1A 4 P3. \\ Richard L. Hall \\ Department of Mathematics and Statistics, Concordia University, \\ 1455 de Maisonneuve Boulevard West, Montréal, Québec, Canada H3G 1M8 \\ Hakan Ciftci \\ Gazi Universitesi, Fen-Edebiyat Fakültesi, Fizik Bölümü, 06500 Teknikokullar, Ankara, Turkey.
}

\begin{abstract}
We apply the Asymptotic Iteration Method to obtain the bound-state energy spectrum for the $d$-dimensional Klein-Gordon equation with scalar $S(r)$ and vector potentials $V(r)$. When $S(r)$ and $V(r)$ are both Coulombic, we obtain all the exact solutions; when the potentials are both of Kratzer type, we obtain all the exact solutions for $S(r)=V(r)$; if $S(r)>V(r)$ we obtain exact solutions under certain constraints on the potential parameters: in this case, a possible general solution is found in terms of a monic polynomial, whose coefficients form a set of elementary symmetric polynomials.
\end{abstract}

PACS: 03.65.w, 03.65.Fd, 03.65.Ge.

\section{INTRODUCTION}

The search for exact solutions of wave equations, whether non-relativistic or relativistic, has been an important research area since the birth of quantum mechanics. Recently the Asymptotic Iteration Method (AIM) has received much attention as a method for solving the Schrödinger equation [1]-[17], both analytically and approximately. It has been applied to a large number of physically interesting potentials and has often yielded highly-accurate results. Very recently, AIM has been used to study the bound states of the Klein-Gordon and Dirac equations for a number of special potentials [18]-[29]. In the case of the Klein-Gordon equation, AIM was used to study the bound-states in the case of equal vector and scalar potential. To our knowledge, the method has never been used to study the boundstates of the Klein-Gordon equation in the case of unequal vector and scalar potentials. In the present paper, we have adapted the method to treat problems where the unequal vector and scalar potentials are of Coulomb or Kratzer type. Our main goal is to investigate the exact solutions, whether the scalar and vector potentials, $S(r)$ and $V(r)$, are equal or not. In the case of equal vector and scalar potentials, it is known that the Klein-Gordon equation usually reduces to a Schrödinger-type equation, which can be studied, for example, by use of the Nikiforon-Uvarov method [30], or by transforming the equation into a classical hypergeometric differential equation with known solution [31]- 32]. It is known [33]- 35] that when $S(r) \geq V(r)$, bound-state solutions exist. Usually the case when the scalar potential is equal to the vector potential is considered separately. The advantage of AIM is that it allows a unified approach that can be used to study the bound-state solutions with equal or unequal scalar and vector potentials.

The Klein Gordon equation has received considerable attention in the literature [35]- 45]. The present paper is organized as follows. Section II is devoted to a brief introduction to the Klein-Gordon equation in $d$-dimensions. In section III we summarize the Asymptotic Iteration Method (AIM) [1]. In Section IV we use AIM to find detailed analytic solutions to the Klein-Gordon equation with Coulomb potentials in $d$-dimensions. The lowest even-parity solution for the Coulomb problem in one dimension is paradoxical and is still under study [46]-[50]. Our solutions in section IV for $d=1$ confirm the exact solutions of the Klein Gordon equation with mixed vector and scalar Coulomb

\footnotetext{
*Electronic address: nsaad@upei.ca

${ }^{\dagger}$ Electronic address: rhall@mathstat.concordia.ca

‡Electronic address: hciftci@gazi.edu.tr
} 
potentials on the half-line obtained earlier by de Castro [48. In Section V, we use AIM to derive exact solutions with equal and unequal scalar and vector Kratzer-type potentials. Our conclusions are presented in section VI.

\section{THE KLEIN-GORDON EQUATION IN $d$ DIMENSIONS}

The $d$-dimensional Klein-Gordon equation for a particle of mass $M$ with radially symmetric Lorentz vector and Lorentz scalar potentials, $V(r)$ and $S(r), r=\|\mathbf{r}\|$, is given (in atomic units $\hbar=c=1$ ) [33, 34] by

$$
\left\{-\Delta_{d}+[M+S(r)]^{2}\right\} \Psi(\mathbf{r})=[E-V(r)]^{2} \Psi(\mathbf{r}),
$$

where $E$ denotes the energy and $\Delta_{d}$ is the $d$-dimensional Laplacian. Transforming to the $d$ dimensional spherical coordinates $\left(r, \theta_{1} \ldots \theta_{D-1}\right)$, the variables can be separated using

$$
\Psi(\mathbf{r})=R(r) Y_{l_{d-1}, \ldots, l_{1}}\left(\theta_{1} \ldots \theta_{d-1}\right)
$$

where $R(r)$ is a radial function, and $Y_{l_{d-1}, \ldots, l_{1}}\left(\theta_{1} \ldots \theta_{d-1}\right)$ is a normalized hyper-spherical harmonic with eigenvalue $l(l+d-2), l=0,1,2, \ldots$ Thus, we obtain the radial equation of Klein-Gordon equation in $d$ dimensions by substituting Eq.(2) into Eq.(11)

$$
-R^{\prime \prime}(r)-\left(\frac{d-1}{r}\right) R^{\prime}(r)+\left\{\frac{l(l+D-2)}{r^{2}}+[M+S(r)]^{2}-[E-V(r)]^{2}\right\} R(\mathbf{r})=0 .
$$

Writing $R$ as $R(r)=r^{-(D-1) / 2} u(r)$ gives the radial equation

$$
-u^{\prime \prime}(r)+\left\{\frac{(k-1)(k-3)}{4 r^{2}}+\left[[M+S(r)]^{2}-[E-V(r)]^{2}\right]\right\} u(r)=0 .
$$

where $k=d+2 l$ and $u(r)$ is the reduced radial wave function satisfying $u(0)=0$.

\section{BRIEF INTRODUCTION OF THE SOLUTION METHOD}

The asymptotic iteration method was introduced [1] to obtain exact and approximate solutions of eigenvalue equations [2]. The first step in applying this method to solve Schrödinger-type equations is to transform these equations, with the aid of appropriate asymptotic forms, to second-order homogeneous linear differential equations of the general form

$$
y^{\prime \prime}=\lambda_{0}(r) y^{\prime}+s_{0}(r) y,
$$

for which $\lambda_{0}(r)$ and $s_{0}(r)$ are functions in $C^{\infty}$. Here primes denotes the derivatives with respect to $r$. A key feature of AIM is to note the invariant structure of the right-hand side of (5) under further differentiation. Indeed, if we differentiate (5) with respect to $r$, we find that

$$
y^{\prime \prime \prime}=\lambda_{1}(r) y^{\prime}+s_{1}(r) y
$$

where

$$
\left\{\begin{array}{l}
\lambda_{1}=\lambda_{0}^{\prime}+s_{0}+\lambda_{0}^{2} \\
s_{1}=s_{0}^{\prime}+s_{0} \lambda_{0}
\end{array}\right.
$$

Meanwhile the second derivative of (5) yields

$$
y^{(4)}=\lambda_{2}(r) y^{\prime}+s_{2}(r) y
$$

for which

$$
\left\{\begin{array}{l}
\lambda_{2}=\lambda_{1}^{\prime}+s_{1}+\lambda_{0} \lambda_{1} \\
s_{2}=s_{1}^{\prime}+s_{0} \lambda_{1}
\end{array}\right.
$$

Thus, for the $(n-1)^{t h}$ and $(n)^{t h}$ derivatives of (5) $, n=1,2, \ldots$, we have

$$
\begin{aligned}
& y^{(n+1)}=\lambda_{n-1}(r) y^{\prime}+s_{n-1}(r) y \\
& y^{(n+2)}=\lambda_{n}(r) y^{\prime}+s_{n}(r) y
\end{aligned}
$$


respectively, where

$$
\left\{\begin{array}{l}
\lambda_{n}=\lambda_{n-1}^{\prime}+s_{n-1}+\lambda_{0} \lambda_{n-1} \\
s_{n}=s_{n-1}^{\prime}+s_{0} \lambda_{n-1}
\end{array}\right.
$$

In an earlier paper [1] we proved the principal theorem of the asymptotic iteration method (AIM), namely

Theorem 1: Given $\lambda_{0}$ and $s_{0}$ in $C^{\infty}$, the differential equation (5) has the general solution

$$
y(r)=\exp \left(-\int^{r} \frac{s_{n-1}}{\lambda_{n-1}} d t\right)\left[C_{2}+C_{1} \int^{r} \exp \left(\int^{t}\left(\lambda_{0}+\frac{2 s_{n-1}}{\lambda_{n-1}}\right) d \tau\right) d t\right]
$$

if for some $n>0$

$$
\delta_{n}=\lambda_{n} s_{n-1}-\lambda_{n-1} s_{n}=0
$$

Recently, it has been shown [51] that the termination condition (11) is necessary and sufficient for the differential equation (5) to have polynomial-type solutions. In the next section, we shall apply this method to obtain the exact solutions for the relativistic $d$-dimension Klein Gordon equation with Coulomb potentials. The results obtained in the next section are known since the Klein-Gordon equation, in this particular case, reduces into an hypergeometric-type differential equation which has known solutions in terms of confluent hypergeometric function. However, the point is to unify the technique of using AIM to obtain analytic solutions, by first studying the known Coulomb case, and then going on to the more-general Kratzer case.

\section{THE KLEIN-GORDON EQUATION WITH COULOMB POTENTIALS IN $d$-DIMENSIONS}

In this section, we use AIM to study the $d$-dimensional Klein-Gordon equation with vector $V(r)$ and scalar $S(r)$ Coulomb potentials [52]-[53], namely

$$
V(r)=-\frac{v}{r}, \quad S(r)=-\frac{s}{r}
$$

In this case, the Klein-Gordon equation (4) reads

$$
-u^{\prime \prime}(r)+\left\{\frac{(k-1)(k-3)+4\left(s^{2}-v^{2}\right)}{4 r^{2}}-\frac{2(M s+E v)}{r}\right\} u(r)=\left(E^{2}-M^{2}\right) u(r) .
$$

In order to solve this Schrödinger-like differential equation using AIM, as mentioned earlier, the first step is to transform (13) into the standard form (5). We note that the differential equation (13) has one regular singular point at $r=0$ and an irregular singular point at $r=\infty$. The asymptotic solution of (13) as $r \rightarrow \infty$ is given by $u(r) \approx e^{-\sqrt{M^{2}-E^{2}} r}$, meanwhile the indicial equation of (13) at the regular singular point $r=0$ yields

$$
c^{2}-c-s^{2}+v^{2}-\frac{1}{4}(k-1)(k-3)=0 .
$$

Thus the exact solution of (13) may assume the form

$$
u(r)=r^{c} e^{b r} f(r)
$$

where $b=-\sqrt{M^{2}-E^{2}}$ and

$$
c=\frac{1}{2}+\sqrt{\left(\frac{k}{2}-1\right)^{2}+s^{2}-v^{2}}
$$

because only the negative root yields a regular wave function at $r=0$. Substituting (14) in (13), we immediately obtain

$$
f^{\prime \prime}(r)=-2\left(\frac{c}{r}+b\right) f^{\prime}(r)+\left(\frac{-2(M s+E v)-2 c b}{r}\right) f(r)
$$


This is a confluent hypergeometric differential equation which has a regular singular point at $r=0$ and an irregular singularity at $r=\infty$. The application of AIM is now initiated with $\lambda_{0}=-2\left(\frac{c}{r}+b\right)$ and $s_{0}=\frac{-2(M s+E v)-2 c b}{r}$, and then terminated with the condition (11). We find

$$
\delta_{n}=0 \quad \text { iff } \quad \prod_{k=0}^{n}(M s+E v+c b+k b)=0
$$

which in turn yields

$$
M s+E v+c b=-n b, \quad n=0,1,2, \ldots
$$

Further, use of (15) yields the eigenvalue equation

$$
\frac{M s+E v}{\sqrt{M^{2}-E^{2}}}=n+\frac{1}{2}+\sqrt{\left(\frac{k}{2}-1\right)^{2}+s^{2}-v^{2}} .
$$

Upon solving (19) for $E$, we obtain

$$
E=M\left(-\frac{s v}{\beta^{2}+v^{2}} \pm \frac{\beta}{\beta^{2}+v^{2}} \sqrt{\beta^{2}+v^{2}-s^{2}}\right)
$$

where $\beta=n+\frac{1}{2}+\sqrt{\left(\frac{k}{2}-1\right)^{2}+s^{2}-v^{2}}$. The un-normalized wave function can be found using (10), namely

$$
f_{n}(r)=\exp \left(-\int^{r} \frac{s_{n-1}(\tau)}{\lambda_{n-1}(\tau)} d \tau\right)
$$

which, after some algebraic computation, yields

$$
f_{n}(r)=(-1)^{n}(c+n)^{n}(2 c)_{n 1} F_{1}\left(-n ; 2 c ; 2 r \sqrt{M^{2}-E^{2}}\right), \quad n=0,1,2, \ldots
$$

up to a multiplicative constant. Here ${ }_{1} F_{1}$ denotes a confluent hypergeometric function [55]

$$
{ }_{1} F_{1}(a ; b ; x)=1+\frac{a}{b} x+\frac{a(a+1)}{b(b+1)} \frac{x^{2}}{2 !}+\cdots+\frac{(a)_{n}}{(b)_{n}} \frac{x^{n}}{n !}+\cdots=\sum_{k=0}^{\infty} \frac{(a)_{k}}{(b)_{k}} \frac{x^{k}}{k !}
$$

and $(a)_{k}=\Gamma(a+k) / \Gamma(a)$. Using equations (14) and (21), we find that the exact solutions of Klein-Gordon equation with Coulomb potential in arbitrary dimension $d$ is given by

$$
u_{n}(r)=C_{n} r^{\frac{1}{2}+\sqrt{\left(\frac{k}{2}-1\right)^{2}+s^{2}-v^{2}}} e^{-\sqrt{M^{2}-E^{2}} r}{ }_{1} F_{1}\left(-n ; 1+\sqrt{(k-2)^{2}+4\left(s^{2}-v^{2}\right)} ; 2 r \sqrt{M^{2}-E^{2}}\right)
$$

The normalization constant $C_{n}$ can be computed by means of $\int_{0}^{\infty}|R(r)|^{2} r^{D-1} d r=1$, where $R(r)=r^{-(D-1) / 2} u(r)$, which, in turn, requires the computation of the definite integral

$$
I_{n m}(\alpha)=\int_{0}^{\infty} \rho^{\alpha} e^{-\rho}{ }_{1} F_{1}(-n ; \alpha ; \rho){ }_{1} F_{1}(-m ; \alpha ; \rho) d \rho
$$

when $n=m$. Since there is some confusion in the notation used in Refs.([52]-[53]) for the computation of the normalization constant $C_{n}$ in (22), we shall present here a detailed computation of the definite integral (24).

Lemma 1: For $\alpha>-1$, we have

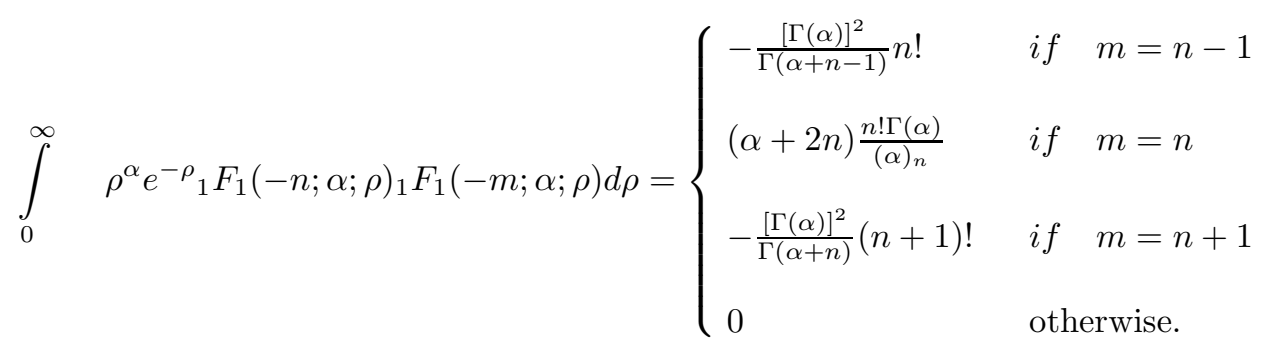


Proof: Using the series representation of the Confluent hypergeometric function (24) and observing that $(-n)_{k}=0$ if $k>n$, we can write (24)

$$
\begin{aligned}
I_{n m} & =\sum_{i=0}^{n} \sum_{k=0}^{m} \frac{(-n)_{i}(-m)_{k}}{(\alpha)_{i}(\alpha)_{k} i ! k !} \int_{0}^{\infty} \rho^{\alpha+i+k} e^{-\rho} d \rho=\sum_{i=0}^{n} \sum_{k=0}^{m} \frac{(-n)_{i}(-m)_{k}}{(\alpha)_{i}(\alpha)_{k} i ! k !} \Gamma(\alpha+i+k+1) \\
& =\sum_{i=0}^{n} \frac{(-n)_{i} \Gamma(\alpha+i+1)}{(\alpha)_{i} i !}{ }_{2} F_{1}(-m, \alpha+i+1 ; \alpha ; 1)=\Gamma(\alpha+1) \sum_{i=0}^{n} \frac{(-n)_{i}(\alpha+1)_{i}(-i-1)_{m}}{(\alpha)_{i} i !}
\end{aligned}
$$

as a consequence [56] of Vandermonde's Theorem ${ }_{2} F_{1}(-n, b ; c ; 1)=(c-b)_{n} /(c)_{n}$. The last sum survives for $m=$ $n-1, n, n+1$. Since we are concerned with the case $m=n$, we consider

$$
\begin{aligned}
I_{n n} & =\frac{\Gamma(\alpha+1)}{(\alpha)_{n}} \sum_{i=0}^{n} \frac{(-n)_{i}(\alpha+1)_{i}(-i-1)_{m}}{(\alpha)_{i} i !} \\
& =\frac{\Gamma(\alpha+1)}{(\alpha)_{n}}\left\{\frac{(-n)_{n-1}(\alpha+1)_{n-1}(-n)_{n}}{(\alpha)_{n-1}(n-1) !}+\frac{(-n)_{n}(\alpha+1)_{n}(-n-1)_{n}}{(\alpha)_{n} n !}\right\}=(\alpha+2 n) \frac{n ! \Gamma(\alpha)}{(\alpha)_{n}}
\end{aligned}
$$

as required. The other cases follow similarly.

Consequently, we have

$$
\int_{0}^{\infty} \rho^{\alpha} e^{-\rho}\left[{ }_{1} F_{1}(-n ; \alpha ; \rho)\right]^{2} d \rho=(\alpha+2 n) \frac{n ! \Gamma(\alpha)}{(\alpha)_{n}}
$$

The normalization constant in (22) is then provided by:

$$
C_{n}^{-2}=\left(\frac{1}{2 \sqrt{M^{2}-E^{2}}}\right)^{2 c+1}(2 c+2 n) \frac{n ! \Gamma(2 c)}{(2 c)_{n}} .
$$

Consequently, the full normalized wave function (23) reads

$$
u_{n}(r)=\sqrt{\frac{\left(2 \sqrt{M^{2}-E^{2}}\right)^{2 c+1}(2 c)_{n}}{2(c+n) n ! \Gamma(2 c)}} r^{c} e^{-\sqrt{M^{2}-E^{2}}} r_{1} F_{1}\left(-n ; 2 c ; 2 r \sqrt{M^{2}-E^{2}}\right),
$$

where $c=\frac{1}{2}+\sqrt{\left(\frac{k}{2}-1\right)^{2}+s^{2}-v^{2}}$. Depending on the values of $s$ and $v$, we may now consider the following three cases:

- If $v=0$, in this case $s$ and $M$ must have the same sign as a result of (20), i.e. $s>0$ (since the right-hand side of (19) is greater than 0). Thus we have

$$
E= \pm M\left(1-\frac{s^{2}}{\left(n+\frac{1}{2}+\sqrt{\left(\frac{k}{2}-1\right)^{2}+s^{2}}\right)^{2}}\right)^{1 / 2}
$$

- If $s=0$, then, again using (19), $E$ and $v$ must have the same sign for $\left(\frac{k}{2}-1\right)^{2}>v^{2}$. For attractive Coulomb potentials $0<v<\frac{D}{2}+l-1$, we have the so-called $\pi$-mesonic atom [54]

$$
E=M\left(1+\frac{v^{2}}{\left(n+\frac{1}{2}+\sqrt{\left(\frac{k}{2}-1\right)^{2}-v^{2}}\right)^{2}}\right)^{-1 / 2}
$$

where $n$ and $l$ are the radial and angular quantum numbers.

- If $s=v>0$, since $|E|<M$, we have

$$
E=M\left(1-\frac{2 v^{2}}{\left(n+\frac{k}{2}-\frac{1}{2}\right)^{2}+v^{2}}\right)
$$




\section{THE KLEIN-GORDON EQUATION WITH KRATZER POTENTIALS IN $d$-DIMENSIONS}

In this section, we consider the scalar and vector potentials with the forms

$$
S(r)=-\frac{s_{1}}{r}+\frac{s_{2}}{r^{2}}, \quad V(r)=-\frac{v_{1}}{r}+\frac{v_{2}}{r^{2}} .
$$

The Klein-Gordon equation (44) then reads

$$
-u^{\prime \prime}+\left\{\frac{-2\left(M s_{1}+E v_{1}\right)}{r}+\frac{2 M s_{2}+s_{1}^{2}+2 E v_{2}-v_{1}^{2}+\frac{1}{4}(k-1)(k-3)}{r^{2}}+\frac{2\left(v_{1} v_{2}-s_{1} s_{2}\right)}{r^{3}}+\frac{s_{2}^{2}-v_{2}^{2}}{r^{4}}\right\} u=\left(E^{2}-M^{2}\right) u .
$$

This differential equation has two irregular singular points at $r=0$ and $r=\infty$. As $r \rightarrow \infty$, the differential equation $u^{\prime \prime} \approx\left(M^{2}-E^{2}\right) u$ has a solution given by $u \approx \exp \left(-\sqrt{M^{2}-E^{2}} r\right)$. As $r \rightarrow 0$, it is clear that the differential equation, after replacing $z=1 / r$ and letting $z \rightarrow \infty$, has a solution $u \approx \exp \left(-\sqrt{s_{2}^{2}-v_{2}^{2}} r^{-1}\right)$. Thus we may assume the exact solution of (32) takes the form

$$
u(r)=e^{-\sqrt{M^{2}-E^{2}} r-\frac{\sqrt{s_{2}^{2}-v_{2}^{2}}}{r}} g(r) .
$$

Substituting this expression in (32), we obtain

$$
\begin{aligned}
g^{\prime \prime}(r) & =-2\left(-\frac{a}{r^{2}}+b\right) g^{\prime}(r)+\left(\frac{-2\left(M s_{1}+E v_{1}\right)}{r}+\frac{2\left(M s_{2}+E v_{2}\right)+s_{1}^{2}-v_{1}^{2}+\frac{1}{4}(k-1)(k-3)+2 a b}{r^{2}}\right. \\
& \left.+\frac{2\left(v_{1} v_{2}-s_{1} s_{2}\right)-2 a}{r^{3}}\right) g(r),
\end{aligned}
$$

where we denote $a=-\sqrt{s_{2}^{2}-v_{2}^{2}}$ and $b=-\sqrt{M^{2}-E^{2}}$.

\section{A. Equal scalar and vector potentials}

In order to solve (34), we consider first the case of equal scalar and vector potentials. i.e. $v_{1}=s_{1}=B$ and $v_{2}=s_{2}=A$. Then, equation (34) reduces to

$$
g^{\prime \prime}(r)=-2 b g^{\prime}(r)+\left(\frac{-2 B(M+E)}{r}+\frac{2 A(M+E)+\frac{1}{4}(k-1)(k-3)}{r^{2}}\right) g(r) .
$$

This equation has a regular singular point at $r=0$ with indicial equation given by $c^{2}-c-2 A(M+E)-\frac{1}{4}(k-1)(k-3)=$ 0 , which implies

$$
c=\frac{1}{2}+\sqrt{\left(\frac{k}{2}-1\right)^{2}+2 A(M+E)},
$$

where we assume $c>0$ (because only the negative root yields a bounded solution at $r=0$.) With $g(r)=r^{c} f(r)$, equation (35) now reads

$$
f^{\prime \prime}(r)=-2\left(\frac{c}{r}+b\right) f^{\prime}(r)+\left(\frac{-2 B(M+E)-2 c b}{r}\right) f(r) .
$$

By a direct application of AIM with $\lambda_{0}=\frac{c}{r}+b$ and $s_{0}=\frac{-2 B(M+E)-2 c b}{r}$, the termination condition (11) implies $\delta_{n}=0, n=0,1,2,3, \ldots$ if and only if

$$
\prod_{k=0}^{n}(B(M+E)+c b+k b)=0, \Rightarrow c+n=\frac{B(M+E)}{\sqrt{M^{2}-E^{2}}} .
$$

Combining (36) and (38), we obtain the eigenvalue equation

$$
\frac{2 B(M+E)}{\sqrt{M^{2}-E^{2}}}=2 n+1+\sqrt{(k-2)^{2}+8 A(M+E)},
$$


which generalizes the results of [32] for the 3-dimension case. Although we can solve Eq.(39) exactly for $E$, the expression for $E$ is rather complicated. We therefore write Eq.(39) as

$$
2 B \sqrt{2 M-\beta^{2}}-\beta\left(2 n+1+\sqrt{(k-2)^{2}+8 A\left(2 M-\beta^{2}\right)}\right)=0
$$

and expand it as a power series in $\beta=\sqrt{M-E}$. Thus we have

$$
2 B \sqrt{2 M}+\left(-2 n-1-\sqrt{(k-2)^{2}+16 A M}\right) \beta-\frac{B}{\sqrt{2 M}} \beta^{2}+\cdots=0 .
$$

As an approximation, if we take only the term in $\beta$, and solve the energy equation, we obtain the non-relativistic energy [57]

$$
E^{\prime}=E-M=-\beta^{2}=-\frac{8 M B^{2}}{\left(2 n+1+\sqrt{(k-2)^{2}+16 A M}\right)^{2}} .
$$

The exact solutions $f_{n}(r)$ of (37) can be obtained using (10) to yield

$$
f_{n}(r)=(-1)^{n}(c+n)^{n}(2 c)_{n 1} F_{1}\left(-n ; 2 c ; 2 \sqrt{M^{2}-E^{2}} r\right)
$$

up to a constant. Consequently, the exact solutions (33) of (32), in the case equal scalar and vector potentials, are given by

$$
u_{n}(r)=N_{n} r^{c} e^{-\sqrt{M^{2}-E^{2}} r} F_{1}\left(-n ; 2 c ; 2 r \sqrt{M^{2}-E^{2}}\right), \quad n=0,1,2, \ldots
$$

where the normalization constant (implied by Lemma 1) now becomes

$$
N_{n}=\sqrt{\frac{\left(2 \sqrt{M^{2}-E^{2}}\right)^{2 c+1}(2 c)_{n}}{2(c+n) n ! \Gamma(2 c)}},
$$

where $c$ is given by (36). We note that in the case $A=0$, equation (39) yields

$$
E=M\left(1-\frac{2 B^{2}}{\left(n+\frac{k}{2}-\frac{1}{2}\right)^{2}+B^{2}}\right),
$$

which is in complete agreement with our results for the Coulomb potential.

\section{B. Unequal scalar and vector potentials}

In the case of different scalar and vector potentials, we have $a \neq 0$ and therefore we have to approach equation (34) directly. The equation, however, has two irregular singular points, at $r=0$ and $r=\infty$, and the standard techniques of solving differential equations cannot be applied. AIM, however, has the advantage that it can be applied directly to obtain exact solutions under certain conditions on the potential parameters of (34). Indeed, with $\lambda_{0}(r)=-2\left(-\frac{a}{r^{2}}+b\right)$ and $s_{0}(r)=\frac{-2\left(M s_{1}+E v_{1}\right)}{r}+\frac{2\left(M s_{2}+E v_{2}\right)+s_{1}^{2}-v_{1}^{2}+\frac{1}{4}(k-1)(k-3)+2 a b}{r^{2}}+\frac{2\left(v_{1} v_{2}-s_{1} s_{2}\right)-2 a}{r^{3}}$, the termination relation (11) yields, for $\delta_{n}=0$, where $n=1,2, \ldots$ is the iteration number, the following conditions

$$
\left\{\begin{array}{l}
2 n b=-2\left(M s_{1}+E v_{1}\right) \\
n(n-1)=2\left(M s_{2}+E v_{2}\right)+s_{1}^{2}-v_{1}^{2}+\frac{1}{4}(k-1)(k-3)+2 a b \\
-2 n a=2\left(v_{1} v_{2}-s_{1} s_{2}\right)-2 a
\end{array}\right.
$$

Furthermore, the exact solutions under the constraints (47), using the AIM expression (10), are given by

$$
g_{n}(r)=r^{n} \Rightarrow u(r)=C_{n} r^{n} e^{-\sqrt{M^{2}-E^{2}} r-\frac{\sqrt{s_{2}^{2}-v_{2}^{2}}}{r}}, \quad n=1,2, \ldots
$$


The normalization constants $C_{n}$ of (48) can be computed by the standard identity [58] (in which $K_{\nu}$ is the modified Bessel function of second kind of order $\nu$ )

$$
\int_{0}^{\infty} r^{\mathcal{C}} \exp \left[-\mathcal{B} r-\frac{\mathcal{A}}{r}\right] d r=2\left(\frac{\mathcal{A}}{\mathcal{B}}\right)^{\frac{\mathcal{C}+1}{2}} K_{-\mathcal{C}-1}(2 \sqrt{\mathcal{A B}}), \quad \mathcal{A}>0, \mathcal{B}>0 .
$$

which implies that

$$
C_{n}^{-2}=2\left(\frac{s_{2}^{2}-v_{2}^{2}}{M^{2}-E^{2}}\right)^{\frac{1+n}{4}} K_{-n-1}\left(2\left(s_{2}^{2}-v_{2}^{2}\right)^{\frac{1}{4}}\left(M^{2}-E^{2}\right)^{\frac{1}{4}}\right) .
$$

That is to say, the exact solutions for the differential equation (34) under the constraints (47) are given by

$$
u_{n}(r)=\left(2\left(\frac{s_{2}^{2}-v_{2}^{2}}{M^{2}-E^{2}}\right)^{\frac{1+n}{4}} K_{-n-1}\left(2\left(s_{2}^{2}-v_{2}^{2}\right)^{\frac{1}{4}}\left(M^{2}-E^{2}\right)^{\frac{1}{4}}\right)\right)^{-\frac{1}{2}} r^{n} e^{-\sqrt{M^{2}-E^{2}} r-\frac{\sqrt{s_{2}^{2}-v_{2}^{2}}}{r}} .
$$

Further, we note that the first and the third equations of (47) yield

$$
\left\{\begin{array}{l}
n=\frac{M s_{1}+E v_{1}}{\sqrt{M^{2}-E^{2}}} \\
n=\frac{v_{1} v_{2}-s_{1} s_{2}}{\sqrt{s_{2}^{2}-v_{2}^{2}}}+1,
\end{array}\right.
$$

This, in turn, inplies

$$
\sqrt{s_{2}^{2}-v_{2}^{2}} \sqrt{M^{2}-E^{2}}=\left(M s_{1}+E v_{1}\right) \sqrt{s_{2}^{2}-v_{2}^{2}}-\left(v_{1} v_{2}-s_{1} s_{2}\right) \sqrt{M^{2}-E^{2}} .
$$

Thus, by mean of the second equation of (47), the eigenvalue equation is

$$
\frac{M s_{1}+E v_{1}}{\sqrt{M^{2}-E^{2}}}=\frac{1}{2}+\sqrt{\left(\frac{k}{2}-1\right)^{2}+2\left(M s_{1}+E v_{1}\right) \sqrt{s_{2}^{2}-v_{2}^{2}}-2\left(v_{1} v_{2}-s_{1} s_{2}\right) \sqrt{M^{2}-E^{2}}+2\left(M s_{2}+E v_{2}\right)+s_{1}^{2}-v_{1}^{2}} .
$$

Some important remarks are in order:

- The exact solutions $u_{n}(r), n=1,2, \ldots$ are nodeless for all $n$. Here, $n$ represents the AIM iteration number. Thus, for all the values of the potential parameters that satisfy (51)-(52), the corresponding solution represents a ground-state wave function. Note that each operator now acts on a different parameter space.

- We notice for $n=1$, the second equation of (51) implies $v_{1} v_{2}=s_{1} s_{2}$ and since $s_{2}>v_{2}$, we must have $v_{1}>s_{1}$ which in turn implies $-s_{1}>-v_{1}$. Thus, the requirement for bound states $S(r)>V(r)$ is satisfied. It is also clear that, this is the case for all $n \geq 1$.

- If $s_{2}=v_{2}=0$, we obtain $u_{0}(r)=C_{0} r^{\frac{1}{2}+\sqrt{\left(\frac{k}{2}-1\right)^{2}+s_{1}^{2}-v_{1}^{2}}} e^{-\sqrt{M^{2}-E^{2}} r}$ and

$$
\frac{M s_{1}+E v_{1}}{\sqrt{M^{2}-E^{2}}}=\frac{1}{2}+\sqrt{\left(\frac{k}{2}-1\right)^{2}+s_{1}^{2}-v_{1}^{2}}
$$

which reduces to the ground state solution described for Coulomb potential as mentioned earlier.

- In the case of a pure scalar potential, i.e. $V(r)=0$, we have $v_{1}=v_{2}=0, S(r)=-\frac{s_{1}}{r}+\frac{s_{2}}{r^{2}}>0$,

$$
u_{0}(r)=C_{0} r^{\frac{1}{2}+\sqrt{\left(\frac{k}{2}-1\right)^{2}+2 M s_{1} s_{2}+2 s_{1} s_{2} \sqrt{M^{2}-E^{2}}+2 M s_{2}+s_{1}^{2}}} e^{-\sqrt{M^{2}-E^{2}} r-\frac{s_{2}}{r}},
$$

and

$$
\frac{M s_{1}}{\sqrt{M^{2}-E^{2}}}=\frac{1}{2}+\left(\left(\frac{k}{2}-1\right)^{2}+2 M s_{1} s_{2}+s_{1} s_{2} \sqrt{M^{2}-E^{2}}+2 M s_{2}+s_{1}^{2}\right)^{1 / 2} .
$$

If we further assume that $s_{2}=0$, we obtain

$$
\frac{M s_{1}}{\sqrt{M^{2}-E^{2}}}=\frac{1}{2}+\left(\left(\frac{k}{2}-1\right)^{2}+s_{1}^{2}\right)^{1 / 2},
$$

which again agrees with the result for the Coulomb potential for this particular case. 
In order to move beyond the ground state solutions, equation (51) suggests that the exact solutions $u(r)$ of (34) take the form

$$
u(r)=r^{c} e^{-\sqrt{M^{2}-E^{2}} r-\frac{\sqrt{s_{2}^{2}-v_{2}^{2}}}{r}} f(r) .
$$

Substituting this expression in (33), we obtain

$$
\begin{aligned}
f^{\prime \prime}(r) & =-2\left(\frac{c}{r}-\frac{a}{r^{2}}+b\right) f^{\prime}(r)+\left(\frac{-2\left(M s_{1}+E v_{1}\right)-2 c b}{r}+\frac{2\left(M s_{2}+E v_{2}\right)+s_{1}^{2}-v_{1}^{2}+\frac{1}{4}(k-1)(k-3)-c^{2}+c+2 a b}{r^{2}}\right. \\
& \left.+\frac{2\left(v_{1} v_{2}-s_{1} s_{2}\right)-2 a+2 c a}{r^{3}}\right) f(r) .
\end{aligned}
$$

where again $a=-\sqrt{s_{2}^{2}-v_{2}^{2}}$ and $b=-\sqrt{M^{2}-E^{2}}$. We may now choose $c$ such that

$$
v_{1} v_{2}-s_{1} s_{2}-a+c a=0 \Rightarrow c=\frac{v_{1} v_{2}-s_{1} s_{2}}{\sqrt{s_{2}^{2}-v_{2}^{2}}}+1 .
$$

which in turn reduces Eq.(56) to

$$
f^{\prime \prime}(r)=-2\left(\frac{c}{r}-\frac{a}{r^{2}}+b\right) f^{\prime}(r)+\left(\frac{-2\left(M s_{1}+E v_{1}\right)-2 c b}{r}+\frac{G}{r^{2}}\right) f(r),
$$

where we denote

$$
G=2\left(M s_{2}+E v_{2}\right)+s_{1}^{2}-v_{1}^{2}+\frac{1}{4}(k-1)(k-3)-c^{2}+c+2 a b .
$$

Although (58) still has an irregular singular point at $r=0$, the direct application of AIM with $\lambda_{0}=-2\left(\frac{c}{r}-\frac{a}{r^{2}}+b\right)$ and $s_{0}=\frac{-2\left(M s_{1}+E v_{1}\right)-2 c b}{r}+\frac{G}{r^{2}}$, implies, by means of the termination condition (11), that

$$
M s_{1}+E v_{1}+c b=-n b \quad \Rightarrow \quad c+n=\frac{M s_{1}+E v_{1}}{\sqrt{M^{2}-E^{2}}}, \quad n=0,1,2, \ldots,
$$

where for $n=0,1,2, \ldots$ the following constraints on $G$ must hold

$$
\begin{aligned}
\mathcal{G}_{0} & :=G=0 \\
\mathcal{G}_{1} & :=G^{2}-2 c G-4 a b=0 \\
\mathcal{G}_{2} & :=G^{3}-2(3 c+1) G^{2}+4\left(2 c^{2}+c-4 b a\right) G+16 a b(2 c+1)=0 \\
\mathcal{G}_{3} & :=G^{4}-4(3 c+2) G^{3}+4\left(11 c^{2}+3+13 c-10 a b\right) G^{2}+\left(192 a b-72 c^{2}+240 c a b-24 c-48 c^{3}\right) G \\
& -144 b a+144 a^{2} b^{2}-432 c a b-288 c^{2} a b=0
\end{aligned}
$$

and so on, for higher iteration numbers.

\section{The case $\mathcal{G}_{0}=0$}

In the case $G=0$, we have

$$
c=\frac{1}{2}+\sqrt{\left(\frac{k}{2}-1\right)^{2}+2 \sqrt{s_{2}^{2}-v_{2}^{2}} \sqrt{M^{2}-E^{2}}+2\left(M s_{2}+E v_{2}\right)+s_{1}^{2}-v_{1}^{2}}
$$

as a root of (59) and, again, $c>0$ because only the negative root yields a regular wave function at $r=0$. On the other hand, equation (60) implies

$$
c=\frac{M s_{1}+E v_{1}}{\sqrt{M^{2}-E^{2}}}
$$


where now (57) gives

$$
\sqrt{s_{2}^{2}-v_{2}^{2}} \sqrt{M^{2}-E^{2}}=\left(M s_{1}+E v_{1}\right) \sqrt{s_{2}^{2}-v_{2}^{2}}-\left(v_{1} v_{2}-s_{1} s_{2}\right) \sqrt{M^{2}-E^{2}} .
$$

Therefore, the ground-state energy equation in $d$-dimensions is given by

$$
\frac{M s_{1}+E v_{1}}{\sqrt{M^{2}-E^{2}}}=\frac{1}{2}+\sqrt{\left(\frac{k}{2}-1\right)^{2}+2\left(M s_{1}+E v_{1}\right) \sqrt{s_{2}^{2}-v_{2}^{2}}-2\left(v_{1} v_{2}-s_{1} s_{2}\right) \sqrt{M^{2}-E^{2}}+2\left(M s_{2}+E v_{2}\right)+s_{1}^{2}-v_{1}^{2}},
$$

which in complete agreement with equation (54).

\section{The case $\mathcal{G}_{1}=0$}

In this case, the constraint $\mathcal{G}_{1}:=G^{2}-2 c G-4 a b=0$ implies $G=c \pm \sqrt{c^{2}+4 a b}$, which implies by (59) for $G>0$, that

$$
\sqrt{c^{2}+4 a b}=2\left(M s_{2}+E v_{2}\right)+s_{1}^{2}-v_{1}^{2}+\frac{1}{4}(k-1)(k-3)-c^{2}+2 a b .
$$

In order to simplify the notation, we denote

$$
\mu=2\left(M s_{2}+E v_{2}\right)+s_{1}^{2}-v_{1}^{2}+\frac{1}{4}(k-1)(k-3)+2 a b
$$

and solve (65) for $c>0$ to obtain

$$
c=\frac{1}{2} \sqrt{2+4 \mu+2 \sqrt{4 \mu+1+16 a b}}
$$

Meanwhile, (57) and (60) imply, for $a b=\sqrt{M^{2}-E^{2}} \sqrt{s_{2}^{2}-v_{2}^{2}}$, that

$$
a b=\frac{1}{2}\left(M s_{1}+E v_{1}\right) \sqrt{s_{2}^{2}-v_{2}^{2}}-\frac{1}{2}\left(v_{1} v_{2}-s_{1} s_{2}\right) \sqrt{M^{2}-E^{2}} .
$$

The eigenenergy is then given, from (67), by the equation

$$
\frac{M s_{1}+E v_{1}}{\sqrt{M^{2}-E^{2}}}=\frac{1}{2} \sqrt{2+4 \mu+2 \sqrt{4 \mu+1+16 a b}}-1
$$

where $\mu$ and $a b$ are given by (66) and (68), respectively. Further, the exact solution of (56) and $G=c+\sqrt{c^{2}+4 a b}$ becomes

$$
f_{1}(r)=r-\frac{2 a}{G}
$$

up to a constant. Thus

$$
u_{1}(r)=C_{1}\left(r-\frac{2 a}{G}\right) r^{c} e^{-\sqrt{M^{2}-E^{2}} r-\frac{\sqrt{s_{2}^{2}-v_{2}^{2}}}{r}},
$$

where the normalization constant $C_{1}$ can be computed by means of (49).

\section{E. The case $\mathcal{G}_{2}=0$}

In this case, we have for $M s_{1}+E v_{1}+c b=-2 b$ that $\mathcal{G}_{2}:=G^{3}-2(3 c+1) G^{2}+4\left(2 c^{2}+c-4 b a\right) G+16 a b(2 c+1)=0$, where $G$ is given by (59). The eigenvalue equation is then given by the root of this equation along with $G$ given by (59) and

$$
a b=\frac{1}{3}\left(M s_{1}+E v_{1}\right) \sqrt{s_{2}^{2}-v_{2}^{2}}-\frac{1}{3}\left(v_{1} v_{2}-s_{1} s_{2}\right) \sqrt{M^{2}-E^{2}},
$$

in a similar fashion to the previous case. After some algebraic computations, we obtain for the exact solution that

$$
f_{2}(r)=\left(r-\frac{G-4 c-2}{4 b}+\frac{1}{4 b} \sqrt{16 a b+8 c+4+4 c G-G^{2}}\right)\left(r-\frac{G-4 c-2}{4 b}-\frac{1}{4 b} \sqrt{16 a b+8 c+4+4 c G-G^{2}}\right) .
$$




\section{F. The general case $\mathcal{G}_{n}:=0$}

The structure of the wave functions for the cases $\mathcal{G}_{0}=0, \mathcal{G}_{1}=0$, and $\mathcal{G}_{2}=0$, etc., allows us to formulate a possible general solution of the Klein-Gordon equation in the case of unequal scalar and vector Kratzer potentials for arbitrary $n$ and $d$. For this purpose, we assume that the general form of the exact solution of the differential equation (59) takes the form of a monic polynomial

$$
f_{n}(r)=\prod_{k=1}^{n}\left(r-\sigma_{k}\right)=\sum_{k=0}^{n} a_{k} r^{k}
$$

whose coefficients $\left\{a_{k}\right\}_{k=0}^{n}$ are the elementary symmetric polynomials [59]

$$
\left\{\begin{aligned}
a_{n} & =1, \\
a_{n-1} & =-\sum_{1 \leq k \leq n} \sigma_{k}, \\
a_{n-2} & =\sum_{1 \leq i<j \leq n} \sigma_{i} \sigma_{j}, \\
a_{n-3} & =-\sum_{1 \leq i<j<k \leq n} \sigma_{i} \sigma_{j} \sigma_{k}, \\
\cdots & =\ldots \sum_{1 \leq j_{1}<j_{2}<\cdots<j_{k} \leq n} \sigma_{j_{1}} \ldots \sigma_{j_{k}} \\
a_{k} & =\ldots \sum_{k=1}^{n} \sigma_{k} \\
a_{0} & =(-1)^{n} \prod_{k=1}^{n}
\end{aligned}\right.
$$

On substituting (174) into the differential equation (158), we find that the coefficients $\left\{a_{k}\right\}_{k=0}^{n}$ must satisfy the following set of linear equations:

$$
\left\{\begin{array}{l}
G a_{0}+2 a a_{1}=0, \\
4 a a_{2}+(G-2 c) a_{1}+2 n b a_{0}=0, \\
{[n(n-1)+2 c n-G] a_{n}=2 b a_{n-1},} \\
2 a(k+3) a_{k+3}+(G-2 c(k+2)-(k+1)(k+2)) a_{k+2}+(F-2 b(k+1)) a_{k+1}=0, \text { for } \quad 0 \leq k \leq n-3 .
\end{array}\right.
$$

In order to understand the application of these formulae, we consider the case of $n=3$ : equation (76) implies

$$
\left\{\begin{array}{l}
a_{2}=-\frac{1}{4 a}\left(G-2 c-\frac{12 a b}{G}\right) a_{1}, \\
a_{3}=\frac{2 b}{6+6 c-G} a_{2}, \\
6 a a_{3}+(G-4 c-2) a_{2}+4 b a_{1}=0, \text { since } \quad k=0 .
\end{array}\right.
$$

The solution of this system, for $a_{1} \neq 0$, gives the condition $\mathcal{G}_{3}$ on $G$ that reads

$$
\begin{aligned}
G^{4} & -4(3 c+2) G^{3}+4\left(11 c^{2}+3+13 c-10 a b\right) G^{2}+\left(192 a b-72 c^{2}+240 c a b-24 c-48 c^{3}\right) G \\
& -144 b a+144 a^{2} b^{2}-432 c a b-288 c^{2} a b=0
\end{aligned}
$$

That is to say, the same condition that we obtain if we apply AIM to (58) with three iterations, i.e $n=3$. Equations (76) are the full set of restrictions on the parameters of the Kratzer potentials (31) in the case of unequal scalar and vector parts.

\section{CONCLUSION}

In this paper, the Klein Gordon equation in arbitrary dimension has been solved exactly for the bound states corresponding to Coulomb or Kratzer scalar $S(r)$ and vector $V(r)$ potentials. When both potentials are Coulombic, equal 
or not, we find all the analytic solutions. When both potentials are of Kratzer type, we find the exact solutions when $S(r)=V(r)$; when the potentials are unequal and the scalar potential dominates, we generate exact solutions under certain specific conditions on the potential parameters. Furthermore, a general solution is found in terms of a monic polynomial whose coefficients form a set of elementary symmetric polynomials. Our method of solution is based on the recently-introduced Asymptotic Iteration Method. This approach has the advantage of simplicity in the exact cases, and flexibility, leading to approximations, when exact solutions are not attainable.

\section{Acknowledgments}

Partial financial support of this work under Grant Nos. GP3438 and GP249507 from the Natural Sciences and Engineering Research Council of Canada is gratefully acknowledged by two of us (respectively [RLH] and [NS]).

[1] Hakan Ciftci, Richard L Hall and Nasser Saad, Asymptotic iteration method for eigenvalue problems, J. Phys. A: Math. Gen. 36 (2003) 11807.

[2] Hakan Ciftci, Richard L Hall and Nasser Saad, Construction of exact solutions to eigenvalue problems by the asymptotic iteration method, J. Phys. A: Math. Gen. 38 (2005) 1147.

[3] Nasser Saad, Richard L. Hall and Hakan Ciftci, Sextic anharmonic oscillators and orthogonal polynomials, J. Phys. A: Math. Gen. 39 (2006) 8477.

[4] Hakan Ciftci, Richard L. Hall, and Nasser Saad, Perturbation theory in a framework of iteration methods, Phys. Lett. A, 340 (2005) 388.

[5] O. Bayrak and I. Boztosun, Arbitrary $\ell$-state solutions of the rotating Morse potential by the asymptotic iteration method, J. Phys. A: Math. Gen. 39 (2006) 6955.

[6] T. Barakat, K. Abodayeh and A. Mukheimer, The asymptotic iteration method for the angular spheroidal eigenvalues J. Phys. A: Math. Gen. 38 (2005) 1299.

[7] T. Barakat, K. Abodayeh, B. Abdallah and O. M. Al-Dossary, The asymptotic iteration method for the angular spheroidal eigenvalues with arbitrary complex size parameter c, Canadian J. Phys. 84 (2006) 121.

[8] T. Barakat, The asymptotic iteration method for the eigenenergies of the Schrödinger equation with the potential $V(r)=$ $-Z / r+g r+\lambda / r^{2}$, J. Phys. A: Math. Gen. 39 (2006) 823.

[9] Francisco M Fernández, On an iteration method for eigenvalue problems J. Phys. A: Math. Gen. 37 (2004) 6173.

[10] Paolo Amore and Francisco M Fernández, Comment on an application of the asymptotic iteration method to a perturbed Coulomb model, J. Phys. A: Math. Gen. 39 (2006) 10491.

[11] O. Bayrak and I. Boztosun, Application of the asymptotic iteration method to the exponential cosine screened Coulomb potential, Int. J. Quant. Chem. 107 (2006) 1040.

[12] K. Abodayeh, T. Barakat and O. M. Al-Dossary, Exact solutions for vibrational levels of the Morse potential via the asymptotic iteration method, Czechoslovak J. Phys. 56 (2006) 583.

[13] O. Bayrak, I. Boztosun and H. Ciftci, Exact Analytical Solutions to the Kratzer Potential by the Asymptotic Iteration Method, Int. J. Quant. Chem. 107 (2007) 540.

[14] O. Bayrak and I. Boztosun, Bound state solutions of the Hulthén potential by using the asymptotic iteration method, Phys. Scr. 76 (2007) 92.

[15] O. M. Al-Dossary, Morse potential eigen-energies through the asymptotic iteration method, Int. J. Quantum Chemistry 107 (2007) 2040.

[16] T. Barakat, K. Abodayeh, Exact solutions for vibrational levels of the Morse potential via the asymptotic iteration method, Czechoslovak J. Phys. 56 (2006) 583.

[17] T. Barakat, The asymptotic iteration method for the eigenenergies of the anharmonic oscillator potential $V(x)=A x^{2 \alpha}+$ $B x^{2}$, Phys. Lett. A 344 (2005) 411.

[18] Hakan Ciftci, Richard L. Hall, and Nasser Saad, Iterative solutions to the Dirac equation, Phys. Rev. A 72 (2005) 022101.

[19] T. Barakat, The asymptotic iteration method for Dirac and Klein-Gordon equations with a linear scalar potential, Int. J. Mod. Phys. A 21 (2006) 4127.

[20] A. Durmus and F. Yasuk, Relativistic and nonrelativistic solutions for diatomic molecules in the presence of double ringshaped Kratzer potential, J. Chem. Phys. 126 (2007) 074108.

[21] Wen-Chao Qiang, Xiao Peng Li and Ai Ping Zhang, New features of an asymptotic iteration method for the Dirac equation and their applications, Phys. Scr. 75 (2007) 29-33.

[22] T. Barakat, The asymptotic iteration method for Dirac and Klein-Gordon equations with a linear scalar potential, Int. J. Mod. Phys. A, 21 (2006) 4127-4136.

[23] I. Boztosun, M. Karakoc, F. Yasuk, and A. Durmus, Asymptotic iteration method solutions to the relativistic DuffinKemmer-Petiau equation, J. Math. Phys. 47 (2006) 062301.

[24] F. Yasuk, A. Durmus, and I. Boztosun, Exact analytical solution to the relativistic Klein-Gordon equation with noncentral equal scalar and vector potentials, J. Math. Phys. 47 (2006) 082302. 
[25] A. Durmus and F. Yasuk, Relativistic and nonrelativistic solutions for diatomic molecules in the presence of double ring-shaped Kratzer potential, J. Chem. Phys. 126 (2007) 74108.

[26] I. Boztosun, F. Taşkın and O. Bayrak Exact Solutions of Klein-Gordon Equation with Exponential Scalar and Vector Potentials, Int. J. Theor. Phys. DOI: 10.1007/s10773-007-9602-0.

[27] O. Bayrak, A. Soylu and I. Boztosun, An approximate solution of Dirac-Hultheén problem with pseudospin and spin symmetry for any $\kappa$ state, J. Math. Phys. 48 (2007) 82302.

[28] A. Durmus and F. Yasuk, Relativistic and nonrelativistic solutions for diatomic molecules in the presence of double ringshaped Kratzer potential J. Chem. Phys. 126 (2007) 074108.

[29] F. Yasuk and A. Durmus, Relativistic solutions for double ring-shaped oscillator potential via asymptotic iteration method, Phys. Scr. 77 (2008) 1.

[30] C. Berkdomir, Relativistic treatment of a spin-zero oarticle subject to a Kratzer-type potential, Am. J. Phys. 75 (2007) 81.

[31] Qiang Wen-Chao, Bound states of the Klein-Gordon and Dirac equations for potential $V(r)=A r^{-2}-B r^{-1}$, Chinese Phys. 12 (2003) 1054-1057.

[32] Q. W. Chao, Bound states of the Klein-Gordon equation for ring-shaped Kratzer-type potential, Chinese Phys. 13 (2004) 575 .

[33] W. Greiner, Relativistic Quantum Mechanics. Wave Equations, 3rd ed. (Springer, Berlin 2000).

[34] A. D. Alhaidari, H Bahlouli and A. Al-Hasan, Dirac and Klein-Gordon equations with equal scalar and vector potentials, Physics Letters A 349 (2006) 87.

[35] F. Domínguez-Adame, Bound states of the Klein-Gordon equation with vector and scalar Hulthén-type potentials, Phys. Lett. A 136 (1989) 175-177.

[36] Lars-Erik Lundberg, Spectral and Scattering Theory for the Klein-Gordon Equation, Commun. math. Phys. 31 (1973) 243-257.

[37] M. Bednar, Algebraic treatment of quantum-mechanical models with modified Coulomb potentials, Annals of Phys., 75 (1973) 305-331.

[38] M. Znojil, On the Klein-Gordon equation with the screened Coulomb potential, Phys. Lett. A, 102 (1984) $289-290$.

[39] O. L. de Lange, An operator analysis for the Schrödinger, Klein-Gordon, and Dirac equations with a Coulomb potential, J. Math. Phys., 30 (1989) 858 - 866.

[40] A. R. Nagalakshmi, B. A. Kagali and V. Sivramkrishna, Bound States of KleinGordon Particles in Scalar Screened Coulomb Potential, Int. J. Mod. Phys. A: Particles \& Fields; Gravitation; Cosmology; Nuclear Physics, 17 (2002) $4793-4799$.

[41] A. R. Nagalakshmi and B. A. Kagali, Spinless particles in screened Coulomb potential, Phys. Lett. A, 296 (2002) $192-197$.

[42] Guo Jian-You, Meng Jie and Xu Fu-Xin, Solution of the Dirac Equation with Special Hulthén Potentials, Chin. Phys. Lett. 20 (2003) 602-604.

[43] M. Langer and C. Tretter, Variational principles for eigenvalues of the KleinGordon equation, J. Math. Phys. 47, 103506 (2006).

[44] A. de Souza Dutraa and G. Chenc, On some classes of exactly-solvable KleinGordon equations, Phys. Lett. A, 349 (2006) 297-301.

[45] Guo Jian-You, Fang Zhou, Guo Feng-Liang and Zhou Jian-Hong, Exact solution of the continuous states for generalized asymmetrical Hartmann potentials under the condition of pseudospin symmetry, Int. J. Mod. Phys. A: Particles \& Fields; Gravitation; Cosmology; Nuclear Physics 22 (2007) 4825-4832.

[46] K. M. Case, Singular potentials Phys. Rev. 80 (1950) 797 - 806.

[47] Dai Xianxi, Jixin Dai and Jiqiong Dai Orthogonality criteria for singular states and the nonexistence of stationary states with even parity for the one-dimensional hydrogen atom, Phys. Rev. A 55 (1997) 2617 - 2624.

[48] A. S. de Castro, KleinGordon particles in mixed vectorscalar inversely linear potentials, Phys. Lett. A 338 (2005) 81-89.

[49] G. Barton, On the 1D Coulomb KleinGordon equation, J. Phys. A: Math. Theor. 40 (2007) 10111031.

[50] R. L. Hall, Solutions to the 1d Klein-Gordon equation with cut-off Coulomb potentials, Phys. Lett. A, 372 (2007) 12 - 15.

[51] N. Saad, R. L. Hall, and H. Ciftci, Criterion for polynomial solutions to a class of linear differential equation of second order, J. Phys. A: Math. Gen. 39 (2006) 13445-13454.

[52] Shi-Hai Dong, Xiao-Yan Gu, Zhong-Qi Ma, and Jiang Yu, The Klein-Gordon equation with a Coulomb potential in D dimensions, Int. J. Mod. Phys. E 13 (2003) 555-565.

[53] Zhong-Qi Ma, Shi-Hai Dong, Ziao-Yan Gu, Jiang Yu, and M. Lozada-Cassou, The Klein-Gordon equation with Coulomb plus Scalar potentials in D dimensions, Int. J. Mod. Phys. E 13 (2004) 597-610.

[54] A. S. Davydov, Quantum Mechanics (Pergamon, New York, 1965).

[55] George E. Andrews, Richard Askey, and Ranjan Roy, Special functions, Cambridge University Press (2001), p. 187.

[56] George E. Andrews, Richard Askey, and Ranjan Roy, Special functions, Cambridge University Press (2001), p. 67, Corollary 2.2.3.

[57] L. D. Landau and L. M. Lifshitz, Quantum Mechanics: Non-Relativistic Theory 3 edition, London, Pergamon Press (1977).

[58] I. S. Gradshteyn and I. M. Ryzhik, Table of integrals, series, and product $6^{\text {th }}$ edition, Academic Press (2000), p. 363, formula 3.471.9.

[59] I. G. Macdonald, Symmetric Functions and Hall Polynomials, $2^{\text {th }}$ edition, Oxford: Clarendon Press (1995). 